\title{
Atuação da equipe de enfermagem no cuidado ao paciente grave
}

\author{
Nursing team care in serious patient care \\ Actuación del equipo de enfermería en el cuidado del paciente grave
}

\author{
Bruna Celin de Carvalho ${ }^{1}$
}

\begin{abstract}
RESUMO
Objetivo: Investigar como deve ser a atuação do profissional de enfermagem nos cuidados com o paciente grave, considerando a relevância da humanização da equipe de enfermagem em unidades de terapia intensiva adulta. Método: Trata-se de uma revisão integrativa da literatura, descritiva, tendo como unidade de análise publicações relacionadas à assistência humanizada de enfermagem direcionada ao paciente crítico. Resultado e Discussão: Conforme as informações apuradas na revisão bibliográfica, pode-se verificar que alguns profissionais da enfermagem não conhecem a política nacional de humanização. No tocante a compreensão a respeito do cuidado humanizado, observou-se que os profissionais são prestativos e o cuidar vem ganhando ênfase, tal como o envolvimento, segurança e acessibilidade. E, que agentes estressores podem ser diminuídos pela enfermagem. Todos os artigos utilizados nessa revisão versavam sobre a dificuldade para a aplicação de um cuidado humanizado aos pacientes graves por parte dos profissionais de enfermagem Conclusão: Com base na revisão bibliográfica procedida, observou-se que alguns profissionais de enfermagem desconhecem a Política Nacional de Humanização, observou-se que os profissionais que demonstram algum grau de conhecimento apresentam dificuldades para implementação desta, contudo acreditam que o cuidado humanizado contribui de maneira significativa na recuperação do paciente.
\end{abstract}

Palavras chave: Assistência de Enfermagem; Humanização; Política de Saúde.

\begin{abstract}
Objective: To investigate how nurses should act in the care of the critically ill patient, considering the relevance of the nursing unit in adult intensive care units. Method: It is an integrative review of the literature, descriptive, to have as a unit of analysis the information related to the humanized care of a therapy directed to the critical patient. Results and Discussion: According to the information found in the bibliographic review, it can be verified that some nursing professionals do not know the national humanization policy. With regard to the understanding of humanized care, it has been observed that professionals are helpful and caring is gaining emphasis, such as involvement, safety and accessibility. And, what stressors can be diminished by nursing. All the articles used in this review dealt with the difficulty of applying humanized care to severe patients by nursing professionals Conclusion: Based on the literature review, it was observed that some nursing professionals are unaware of the National Humanization Policy, it was observed that professionals who demonstrate some degree of knowledge present difficulties to implement this, however, believe that humanized care contributes significantly to patient recovery.
\end{abstract}

Key words: Nursing Care; Humanization; Health Policy.

${ }^{1}$ Graduação de Enfermagem, Centro Universitário de Caratinga (UNEC). E-mail: brunacelin@hotmail.com 


\section{RESUMEN}

Objetivo: Investigar cómo debe ser una actuación del enfermero en los cuidados con el paciente grave, considerando la relevancia de la unidad de enfermería en unidades de terapia intensiva adulta. Método: Se trata de una revisión integrativa de la literatura, descriptiva, tener como unidad de análisis las informaciones relacionadas a la asistencia humanizada de una terapia dirigida al paciente crítico. Resultado y Discusión: Conforme a las informaciones constatadas en la revisión bibliográfica, se puede verificar que algunos profesionales de la enfermería no conocen la política nacional de humanización. En cuanto a la comprensión acerca del cuidado humanizado, se observó que los profesionales son provechosos y el cuidar viene ganando énfasis, tal como la implicación, seguridad y accesibilidad. Y, qué agentes estresores pueden ser disminuidos por la enfermería. Todos los artículos utilizados en esta revisión versaban sobre la dificultad para la aplicación de un cuidado humanizado a los pacientes graves por parte de los profesionales de enfermería Conclusión: Con base en la revisión bibliográfica procedida, se observó que algunos profesionales de enfermería desconocen la Política Nacional de Humanización, se observó que los profesionales que demuestran algún grado de conocimiento presentan dificultades para su implementación, sin embargo creen que el cuidado humanizado contribuye de manera significativa en la recuperación del paciente.

Palabras clave: Asistencia de enfermería; humanización; Política de Salud.

\section{INTRODUÇÃO}

Enfermagem é o equivalente a cuidar, tornando imprescindível que o cuidado e a assistência prestados sejam efetivos de maneira humanizada (FONSECA et al., 2013). Essa perspectiva do cuidar de forma humanizada, certamente é uma das mais difíceis de se colocar em prática. A literatura relata que a rotina diária intricada que engloba cenários de unidades críticas, não raramente leva o profissional de enfermagem a prestar assistência de maneira inapropriada, deixando de escutar o paciente de maneira integral e não tratando de suas necessidades elementares (FARIAS et al., 2013).

O cuidado humanizado vem sendo debatido, sobretudo com a política nacional de humanização (PNH) do Ministério da saúde, com o proposito de aplicar os conceitos do sistema único de saúde (SUS) na rotina dos serviços de saúde, guardando a privacidade e proporcionando uma atmosfera receptiva e aconchegante (FONSECA, et al., 2013).

Segundo o estudo de Pott et al, (2013) o cuidar não se limita ao executar de práticas técnicas, mas engloba o paciente integralmente, com suas histórias, emoções e esperanças. É reaver o valor das questões emocionais, psicológicas e físicas. É observar o que o paciente quer emitir, tranquilizar, amparar e valorizalo em todas suas perspectivas.

Humanizar o atendimento na saúde requer dar suporte partindo da integridade ética da palavra, do respeito, reconhecimento e da solidariedade, conferindo um cuidar integral. Neste patamar a humanização do cuidado é uma necessidade que requer ponderar a maneira como tem sido realizada, sobretudo no tocante aos serviços prestados pela equipe de enfermagem na atenção aos pacientes graves (VIANA et al, 2012).

A virtude desse cuidado decorre de conhecimentos e aptidões técnicas além da interação e dialogo dos profissionais com o paciente. Desta forma, o cuidado é prestado junto e para o próximo e, assim, o diálogo é imprescindível no cuidado dos que participam do decurso da hospitalização, sobretudo pacientes graves (POTT et al, 2013). Dessa maneira os cuidados prestados pelo profissional de enfermagem perpassam a assistência prestada, devem ser particulares e imprescindíveis para propiciar ao paciente melhor qualidade de vida e de assistência, ofertando ao paciente melhor responsividade ao tratamento.

Mediante o exposto, o presente trabalho se propõe a investigar como deve ser a atuação do profissional de enfermagem nos cuidados com o paciente grave. O objetivo geral é investigar como deve ser a atuação do profissional de enfermagem nos cuidados com o paciente grave; e os específicos são caracterizar quais pacientes são considerados graves, contextualizando o ambiente em que ficam hospitalizados (UTIs); e descrever o papel do profissional de enfermagem nos cuidados ao paciente grave, a percepção dos familiares, e a contribuição das instituições de formação profissional sobre o tema. 


\section{METODOLOGIA}

Trata-se de um estudo de revisão integrativa, que consiste na construção de uma análise ampla da literatura, que pode contribuir para discussões sobre métodos e resultados de pesquisas, assim como reflexões sobre a realização de futuros estudos. Broome (2000) explica que o propósito deste método de pesquisa é obter um profundo entendimento de um determinado fenômeno baseando-se em estudos anteriores.

Para a busca foram acessados os artigos indexados nas bases de dados SciELO, e LILACS. A revisão compreende o período de julho a setembro de 2016. Para isso utilizou-se os descritores: Assistência de Enfermagem, Humanização, Política de Saúde. Dessa forma foi possível encontrar 19 artigos que atendiam aos critérios de inclusão, que compreendiam ter relação com a questão do cuidado humanizado priorizando o paciente critico, tendo como fonte de informações publicações compondo-se de textos na íntegra, disponíveis em português e de acesso público.

\section{REVISÃO DE LITERATURA}

As Unidades de Terapia Intensiva (UTI's) apareceram no Brasil na década de 70 do século XX, em pleno ápice do desenvolvimento econômico que, nessa conjuntura, favorecia um modelo econômico centralizador de renda e tinha política voltada para a modernização e para desenvolvimento, o que impactou a área da saúde que se encontrava em crescimento, por intermédio do estado (TRANQUITELLI, 2007).

Tranquitelli, (2007) diz que conforme a ênfase assistencial destinada aos problemas na esfera de abrangência individual, entende-se que as unidades de cuidados devam ser ajustadas às necessidades do público atendido fazendo-se de apoio para o estabelecimento de uma assistência eficaz ao paciente internado, sobretudo nas UTIs, por conta de sua peculiaridade: Conceitualmente, verifica-se que a Unidade de Terapia Intensiva destina-se ao atendimento de pacientes em estado agudo ou crítico, mas recuperável, que requerem assistência médica e de enfermagem permanente e especializada. São pacientes sujeitos à instabilidade de funções vitais, que necessitam do apoio de equipamentos especiais de diagnóstico e tratamento(CAR, 1986).

Silva et al (2011), alega que a humanização em UTI pode ser praticada de diversas formas, desde dialogar, esclarecer os procedimentos ao paciente na abordagem com os familiares, respeitando as convicções, princípios e estimulando a criação de regras e rotinas a serem seguidas, como horário de banho, horário para dormir, para que o paciente seja capaz de perceber se está de dia ou de noite etc. A humanização engloba planejamento e supervisão e para isso deve-se instituir normas para que o desempenho de todo sistema de humanização tenha sucesso. O paciente hospitalizado na UTI demanda cuidados de excelência, voltados não somente para os problemas de saúde mas também para os pleitos psicossociais, ambientais e familiares que compõem o quadro de patologia física bio-psico-sócio-espiritual (SILVA, et al, 2011).

A UTI possui alguns atributos específicos, como o convívio diário entre os profissionais e pacientes com as circunstancias de perigo, o destaque no conhecimento técnico-cientifico e no aparato tecnológico para o atendimento biológico, a inquietação dos pacientes e familiares e da equipe de saúde, as rotinas severas e árduas, e a ligeireza de ação no atendimento (NASCIMENTO e TRENTINI, 2004). Considerando este cenário, as publicações sinalizam a necessidade de os profissionais possuírem peculiaridades distintas, afim de prestarem o cuidado da melhor maneira, mesmo com todos empecilhos desse ambiente de trabalho (SILVA e FERREIRA, 2009).

Sobre a utilização de ferramentas tecnológicas em prejuízo do cuidado humanizado, estudos têm comprovado que a utilização de maneira inapropriada e o não comprometimento de alguns profissionais cooperam para tornar a assistência automática, criando um espaço entre o paciente e família da equipe de saúde, desvirtuando o cuidado como ação humana. O profissional de enfermagem na UTI, não raro, deixa de tocar, falar e escutar a pessoas que está a sua frente, absorvido pelo cotidiano árduo e pela prestação de serviço de alta tecnologia (VILA e ROSSI, 2002). 
A equipe de saúde tem dificuldade de diferir o ser humano do maquinário, responsável pela sua sobrevivência, transformando sua relação com a maquina e o cuidado de enfermagem numa conduta mecânica, enxergando o paciente como uma simples continuidade dos aparelhos. Os autores salientam que, os aparelhos oportunizam o pronto atendimento, conferem segurança para a equipe da UTI, contudo podem cooperar para deixar as relações humanas mais apartadas, gerando no paciente e em seus familiares o sentimento de desamparo (NASCIMENTO e ERDMANN, 2006).

Cuidar é um ato repleto de atenção, zelo e desvelo. Simboliza ocupar-se, preocupar-se e sentir afeto pelo próximo. Por "cuidar" entende-se o estar atento integral e permanentemente ao paciente sem negligenciar o fato de que é uma pessoa, um ser único e insubstituível. Dessa forma, esse é o cerne das atenções de quem presta cuidados e, portanto, todas as perspectivas de qualquer natureza são observadas e integram a assistência (CASTRO et al, 2010). No intento de empregar o conceito do autor de cuidar no ambiente de UTI, nota-se a necessidade de um preparo mais eficaz de toda equipe de profissionais da saúde para melhor atender os pacientes. Humanizar os cuidados engloba respeitar a individualidade das pessoas e "conceber um espaço nas instituições de saúde que valide o humano dos indivíduos envolvidos". Dessa forma, para cuidar de maneira humanizada, o profissional de saúde, sobretudo o enfermeiro, que presta cuidados diretamente ao paciente, deve ter a habilidade de compreender a si próprio e ao outro, canalizando esse conhecimento em forma de ação e tendo consciência dos princípios que orientam essa ação. Neste cenário, o respeito ao paciente é elementar em relação aos cuidados humanizados (BARBOSA e SILVA, 2007).

O paciente é antes de tudo um ser humano, ímpar e singular. Sendo o mesmo o cerne dos cuidados, devendo ser atendido em todos pontos de vista. Para isso, é relevante que o profissional esteja apto a escutar, comunicar e compreender o paciente, em prol do cuidado humanizado. Contudo, frequentemente a realidade destoa disso, os profissionais enfrentam diversas burocracias e tecnologias que tomam tempo e atrapalha a assistência humanizada (BARBOSA e SILVA, 2007).

Os profissionais de enfermagem devem ser orientados a amortecer suas emoções, afim de cuidar dos pacientes graves, para que não levem consigo os preconceitos, valores e significados culturais. Portanto, cuidar de quem cuida é primordial para tratar de forma terapêutica dos outros (VILA e ROSSI, 2002).

Essa é a concepção do humanizar idealizado. Tendo empatia, o profissional passa a cuidar, observando o conceito de humanizar que engloba respeito, dignidade, contemplando a locução amar o próximo como a si mesmo (VILA e ROSSI, 2002).

Das informações correlatas à temática da pesquisa, foram reconhecidos: cuidado humanizado ligado a concepção do enfermeiro, da compreensão dos pacientes e familiares e a colaboração acadêmica para a assimilação das práticas humanizadas. Desta forma os resultados serão apresentados de acordo com estas perspectivas.

\section{O Papel Do Enfermeiro No Cuidado Humanizado}

Conforme as informações apuradas pode-se verificar que alguns profissionais da enfermagem não conhecem a politica nacional de humanização. Isso pode ser observado por meio dos estudos, como no de Sanches, et al (2016), onde evidenciou-se que a equipe de saúde demonstra possuir barreiras no tocante a expressão da conceituação de humanização. A concepção de humanização, do ponto de vista de alguns enfermeiros, não pode se limitar ao cenário saúde $X$ enfermidade, porque engloba o cuidado ao ser humano com inúmeras possibilidades a serem descobertas (VIANA et al, 2012). A noção dos enfermeiros a respeito da humanização exibiu algumas diferenças nas respostas, retomando a polissemia da expressão, citada previamente. As percepções foram inconsistentes e referiram inclusive fatos concernentes à atitude do profissional frente ao paciente, os procedimentos que devem ser empregados na assistência em saúde em consentimento com deliberações da $\mathrm{PNH}$, até a deficiência na formação profissional para entendimento e evolução desta concepção (MONGIOVI et al, 2014).

A expressão humanização, na ultima década, foi presente nos conteúdos do ministério e secretarias de estado e municipais de saúde, igualmente nas obras da área da saúde, como um apanhado de medidas que se propõe a qualificação da atenção ao indivíduo enfermo. Tem natureza polissêmica por pertencer a 
movimentos, concepções, atividades de diversas fontes históricas e vertentes de entendimento subordinadas a diversas possibilidades de leitura (SANCHES et al, 2016). Nessa perspectiva, para alguns estudiosos (CASSIANO et al, 2015), a humanização perpassa as fronteiras do agir em saúde, pois possibilita alterações na administração e nos conceitos e princípios contidos na estrutura social, física e funcional, ordenados em conjunto ao sistema de saúde. Assim, é imprescindível que seu entendimento seja atrelado com os temas sociais, políticos, econômicos e culturais.

\section{Compreensão Dos Pacientes E Suas Famílias Sobre Humanização}

O cuidado é uma característica da enfermagem, sendo esse uma prerrogativa para existência do ser humano. Humanização na área da saúde vai além da competência técnocientifica - política dos profissionais, contempla o desenvolvimento da competência das relações interpessoais que devem se fundamentar no respeito ao ser humano, no respeito à vida, na solidariedade, na sensibilidade de percepção das necessidades particulares dos sujeitos envolvidos o cuidado de enfermagem é um cuidado ético e para tal implica o uso de habilidades técnicas, conhecimentos e relação com o outro(FONSECA et al., 2013). No estudo foi explicitado que boa parte dos pacientes se queixam de barulhos frequentes, ruídos e luzes são elementos existentes na unidade intensiva coronariana (FONSECA et al., 2013). Em outra pesquisa, identificou-se circunstâncias consideradas desumanas tais como comentários inconvenientes, falta de privacidade do paciente, uso de apelidos ao menciona-los, em diversos setores, não relegando a tecnologia a exclusividade da desumanização na assistência de enfermagem ao paciente em ambientes onde seu uso é frequente (SILVA et al, 2012).

No tocante a compreensão a respeito do cuidado humanizado, observou-se que os profissionais são prestativos e o cuidar vem ganhando ênfase, tal como o envolvimento, segurança e acessibilidade. $E$, que estes estressores podem ser diminuídos pela enfermagem (FONSECA et al., 2013). Porém, em outra pesquisa analisada, foi averiguado que os familiares exprimem expectativas positivas concernentes à internação do paciente, por meio do acolhimento e informações dadas, presumindo que com as referências e acolhimento da enfermagem, eles poderão superar suas expectativas (SILVA; SANTOS, 2010). Outra pesquisa atestou o valor da escuta, observação e consideração à subjetividade do individuo como fatores elementares que exprimem a humanização no cuidado, apontando que a relação entre a equipe de saúde e a família deve ser uma confluência de subjetividades onde surgem novos entendimentos e interpelações, cooperando para a dinâmica saúde-doença. E, que o respeito aos pacientes e seus familiares é reconhecido como um valor elementar na profissão enfermagem.( CHERNICHARO et al., 2011).

\section{Participação Das Instituições De Ensino Nas Práticas De Humanização}

Em seu estudo, Silva et al, (2011), comprovaram a necessidade de aprofundamento na questão humanização no cuidado, sobretudo no âmbito do ensino e da formação profissional, dado a mesma relevância que o mesmo possui no campo da política pública, economia, cultura, ética, e da formação profissional. Segundo Guilherme Correa Barbosa et al (2013), a política nacional de humanização intenta formar profissionais que em sua performance formulem atividades de eficácia técnica e cientifica, com atitude ética, mas que observem a necessidade e individualidade de cada paciente, com ciência de que este convívio é originador de aperfeiçoamento nas práticas de saúde.

Barbosa et al. (2013) ao proceder revisão integrativa abordando a política nacional de humanização e a formação de profissionais da saúde, concluiu que "a formação dos alunos de graduação em enfermagem e medicina encontra como obstáculo o pluralismo do termo humanização, sem que se valorize o documento do Ministério da Saúde sobre Política Nacional de Humanização". Para o autor é preciso que haja harmonização entre o conteúdo ensinado das ciências humanas com os conteúdos da prática clínica, e que as instituições e locais de formação propiciem o compartilhar dos conhecimentos adquiridos em locais de prestação de serviços e dos ensinamentos inerentes à formação, para que se forjem relações entre os professores, alunos e profissionais da área da saúde. (Barbosa et al (2013).

Para os alunos, a humanização, se conhece e assimila na prática, mas essa experiencia sofre interferência das instituições de ensino, por conta do processo de ensino e aprendizagem provido na formação. Ademais, 
há barreiras a serem superadas no cenário da formação, quando a dinâmica ensino-aprendizagem ocorre de uma maneira e o cumprimento nos ambientes de prática de outra, sem que haja vinculo entre o que aprende em sala de aula com o que se realiza na prática (FREITAS, FERREIRA, 2016). Desta maneira, as pesquisas (BARBOSA et al, 2013; FREITAS, FERREIRA, 2016) fortificam que a relevância de conectar teoria e prática é essencial para humanização, mas esta não se concretiza nas experiencias de aprendizagem. $O$ desempenho do professor e o trabalho em equipe são fatores que inspiram a humanização.

Todos os artigos utilizados possuíam em suas discussões, um assunto recorrente, isto é, versavam sobre a dificuldade para a aplicação de um cuidado humanizado aos pacientes graves por parte dos profissionais de enfermagem. Assim, compreende-se a importância de buscar sempre mais afundo o porquê da falta de prática desse cuidado com a finalidade de distinguir as razões. Assim, os profissionais e gestores terão base para estabelecerem estratégias para minimizarem as consequências dessa falta, e cooperarem para a prestação de uma assistência integral voltada para a qualidade do cuidar.

\section{CONSIDERAÇÕES FINAIS}

Mediante a revisão dos artigos, notou-se que há profissionais de enfermagem que não tem ciência da política nacional de humanização. No Brasil ainda é pequena a discussão de políticas de intervenção considerando os aspectos de humanização. A dimensão subjetiva da qualidade da atenção, como o conforto, o acolhimento e a satisfação dos usuários é algo que parece ainda um discurso ideal e dessemelhante do real. Diversas pesquisas associaram essas dificuldades à falta de uma reflexão mais profunda a respeito da humanização na saúde, desde o processo de formação profissional. A política nacional de humanização preconiza a necessidade de formação de profissionais que sejam aptos em suas rotinas diárias a proceder suas práticas pautados pela eficácia técnica, cientifica e humana, mantendo uma conduta ética, resguardando as necessidades particulares do paciente e que possua uma visão ampla e integral.

A enfermagem deve retomar, no seu desempenhar, essa importância, de cuidado humanizado, englobando o respeito e a dignidade do individuo com seus direitos em todas as etapas da vida, empregando estratégias de comunicação conferindo aconchego e acalento. Observou-se ainda a importância da instrução em prol da humanização no cuidado ao paciente em circunstancias graves de maneira minuciosa desde a Graduação ao longo de toda sua formação, priorizando principalmente, um processo de desenvolvimento de habilidades ético-morais que pautem suas futuras práticas. sugere-se que mais pesquisas sejam realizadas abordando o assunto, para que os profissionais da enfermagem reflitam a respeito da importância de ações do cuidado integral, dando atenção as nuances e subjetividades do ser humano e da família.

\section{REFERÊNCIAS}

1. BARBOSA IA, SILVA, MJP. Cuidado humanizado de enfermagem: o agir com respeito em um hospital universitário. Revista Brasileira de Enfermagem. 2007 Set-Out; 60 (5): 546-51.

2. BROOME, Marion E. Integrative literature reviews for the development of concepts. Concept development in nursing: foundations, techniques and applications. Philadelphia: WB Saunders Company, p. 231-50, 2000.

3. CASSIANO AN, ARAUJO MG, HOLANDA CSM et al. Percepção de enfermeiros sobre a humanização na assistência de enfermagem no puerpério imediato. J. res.: fundam. care. online 2015. jan./mar. 7(1):2051-2060.

4. CASTRO PG. et al. A importância do enfermeiro na promoção da humanização na unidade de terapia intensiva. Revista Eletrônica de Enfermagem do Centro de Estudos de Enfermagem e Nutrição. Jan-jul 1(1): 1-16. 2010.

5. CHERNICHARO IM, SILVA FD, FERREIRA MA. Humanização no cuidado de enfermagem nas concepções de profissionais de enfermagem. Esc. Anna Nery; 2011 out-dez; 15 (4):686-693.

6. FARIAS FBB, VIDAI LL, FARIAS RAR et al. Cuidado humanizado em uti: desafios na visão dos profissionais de saúde. J. res.: fundam. care. online 2013. out./dez. 5(4):635-42.

7. FONSECA GGP, PARCIANELLO MK, ZAMBERLAN C. Agentes estressores em unidade de tratamento intensivo coronariana e o cuidado humanizado de enfermagem. R. Enferm. Cent. O. Min. 2013 mai-ago; 3(2):654-661.

8. FREITAS FDS, FERREIRA MA. Saberes de estudantes de enfermagem sobre a humanização. Rev Bras Enferm [Internet]. 2016 mar-abr;69(2):282-9.

9. GUILHERME CORREA BARBOSA GG, MENEGUIM S, LIMA SAM, MORENO V. Política Nacional de Humanização e formação dos profissionais de saúde: revisão integrativa. Rev. bras. Enferm. 2013 jan-fev; 66(1): 123-7.

10. MENDES KDS, SILVEIRA RCCP, GALVÃO CM. Revisão integrativa: método de pesquisa para a incorporação de evidências na saúde e na enfermagem. Texto Contexto Enferm. 2008 Out-Dez; 17(4): 758-64. 
11. MONGIOVI VG, ANJOS RCCBL, SOARES SBH et al. Reflexões conceituais sobre humanização da saúde: concepção de enfermeiros de Unidades de Terapia Intensiva. Rev Bras Enferm. 2014 mar-abr; 67(2): 306-11.

12. NASCIMENTO ERP, TRENTINI M. O cuidado de enfermagem na unidade de terapia intensiva (UTI): teoria humanística de Paterson e Zderad. Rev Latino-am Enfermagem. 2004; 12 (2): 250-7

13. NASCIMENTO KC, ERDMANN, AL. Cuidado transpessoal de enfermagem a seres humanos em unidade crítica. R Enferm UERJ. 2006; 14 (3): 333-41.

14. POTT FS, STAHLHOEFER T, FELIX JVC, MEIER MJ. Medidas de conforto e comunicação nas ações de cuidado de enfermagem ao paciente crítico. Rev Bras Enferm. 2013 mar-abr; 66(2): 174-9.

15. SANCHES RCN, GERHARDT PC, RÊGO AS, et al. Percepções de profissionais de saúde sobre a humanização em unidade de terapia intensiva adulto. Esc Anna Nery 2016;20(1):48-54.

16. SILVA FD, CHERNICHARO IM, FERREIRA MA. Humanização e desumanização: a dialética expressa no discurso de docentes de enfermagem sobre cuidado. Esc Anna Nery (impr.)2011 abr-jun; 15 (2):306-313.

17. SILVA FD, CHERNICHARO IM, SILVA RC, et al. Discursos de Enfermeiros Sobre Humanização Na Unidade De Terapia Intensiva. Esc Anna Nery (impr.)2012 out - dez; 16 (4):719- 727.

18. SILVA FS, SANTOS I. Estudo Sociopoético sobre as expectativas de familiares em UTI familiares em UTI. Esc Anna Nery Rev Enferm 2010 abr-jun; 14 (2): 230-235.

19. SILVA RC, FERREIRA MA. Representações sociais dos enfermeiros sobre a tecnologia no ambiente da terapia intensiva. Texto Contexto Enferm. 2009; 18 (3): 489-97.

20. SILVA SP; et al. Humanização da assistência atribuída aos profissionais da equipe de enfermagem que atuam em unidades de terapia intensiva. II Congresso de Humanização. I jornada Interdisciplinar de Humanização. Curitiba. 2011.

21. SILVA YB. Análise dos registros de enfermagem sobre dor e analgesia em doentes hospitalizados. Dissertação. São Paulo. Escola de Enfermagem da Universidade de São Paulo. 2001.

22. TRANQUILELLI AM, CIAMPONE MHT. Número de horas de cuidados de enfermagem em unidade de terapia intensiva de adultos. Resumen. Rev. Esc. Enferm. USP. 2007. 41 (3): 371-7. Acesso em: 25 mar. 2018. Disponível em:< http://www.ee.usp.br/reeusp/>

23. VIANA CAMPOS ACV, DUTRA BS, BORGES CM, et al. O cuidado humanizado sob a percepção dos enfermeiros. Enferm. Ver. Belo Horizonte. 2012 mai; 15(1): 47-57.

24. VILA VSC. O significado cultural do cuidado humanizado em unidade de terapia intensiva: muito falado e pouco vivido. Revista LatinoAmericana de Enfermagem. Ribeirão Preto. São Paulo. Mar/Apr. 2002.. 